IPM/P-2006/033

July 6, 2018

\title{
Reconciling large CP-violating phases with bounds on the electric dipole moments in the MSSM
}

\author{
Seyed Yaser Ayazi and Yasaman Farzan \\ Institute for Studies in Theoretical Physics and Mathematics (IPM) \\ P.O. Box 19395-5531, Tehran, Iran
}

\begin{abstract}
The possibility of cancelation between different contributions to $d_{e}, d_{n}$ and $d_{H g}$ has been reconsidered with special emphasis on the region that is phenomenologically interesting (intermediate values of $\tan \beta$ and sub-TeV sfermion masses). It is found that in the range favored by electroweak baryogenesis (i.e., $|\mu| \simeq M_{1}$ or $|\mu| \simeq M_{2}$ ), $\sin \left[\theta_{\mu}+\theta_{M_{1}}\right] \sim 1$ can be compatible with the EDM bounds even for slepton masses below $500 \mathrm{GeV}$. Such large values of the phases promise a successful electroweak baryogenesis. The possibility of large CP-odd effects at linear collider has also been discussed.
\end{abstract}

\section{Introduction}

Elementary particles can possess Electric Dipole Moments (EDMs), only if the CP symmetry is violated. For this reason, studying EDMs of the elementary particles is of prime importance as it can teach us about CPviolation which is closely related to the creation of the baryon asymmetry of the universe. It is well-known that within the Standard Model (SM) of the elementary particles violation of $\mathrm{CP}$ can take place. In fact in the Kaon and B-meson sector, the CP symmetry has been observed to be violated in accordance with the prediction of the Standard Model. However, the maximum possible values of EDMs in the context of SM are extremely small; 
the SM predicts $d_{e} \sim 10^{-38} e \mathrm{~cm}$ [1] and the prediction of SM for $d_{n}$ ranges from $10^{-31} e \mathrm{~cm}$ to $10^{-33} e \mathrm{~cm}[2]$. So far no electric dipole moment for the electron or neutron has been detected but strong bounds on these quantities have been obtained $[3,4,5]$

$$
\left|d_{e}\right|<1.4 \times 10^{-27} \text { e cm } \quad\left|d_{n}\right|<3.0 \times 10^{-26} \text { e cm. }
$$

There are good prospects of improving these bounds by several orders of magnitude in the next few years [6]. Values of EDMs much larger than the SM prediction would indicate new sources of CP-violation with origin in physics beyond the SM.

The Minimal Supersymmetric Standard Model (MSSM) is arguably the most popular model beyond the SM. The general MSSM introduces 44 sources of CP-violation [7]. Mainly for the sake of simplicity, studies in the literature are concentrated on the mSUGRA model (which is also called constrained MSSM or cMSSM) which assumes that at the GUT scale the masses of scalar components of the chiral superfields are unified to $m_{0}$ while the masses of gauginos are also unified to $m_{1 / 2}$. In this model, the A-terms (the trilinear scalar terms in the soft supersymmetry breaking potential) are also universal and set to be proportional to the corresponding Yukawa couplings: at the GUT scale, $A_{\ell}=a_{0} Y_{\ell}, A_{u}=a_{0} Y_{u}$ and $A_{d}=a_{0} Y_{d}$. In the constrained MSSM the number of independent $\mathrm{CP}$-violating phases are reduced to two which are usually attributed to the phases of $a_{0}$ and the mu-term (bilinear Higgs mass term in the superpotential). Taking the values of the parameters at their phenomenologically favorable range $\left(m_{1 / 2} \sim m_{0} \sim 100 \mathrm{GeV}, \tan \beta \sim\right.$ 10, $\theta_{\mu} \sim 1$ and $\theta_{A_{e}} \sim 1$ ), one finds that the EDMs of the electron, neutron and mercury exceed the experimental bounds by several orders of magnitude. In principle, to suppress the EDMs to below their experimental bounds, three possibilities exist:

- The first generation of sleptons and the first two generation of squarks 
are very heavy [8]. This means the production and study of these particles at ILC and LHC will be difficult, if possible at all. The other reason that this possibility is in disfavor is that, with large sfermion masses the annihilation rate of the Lightest Supersymmetric Particle (LSP) may be too low and as a result the relic density of the LSP may be larger than the observed dark matter density.

- The phases of $\mu$ and $a_{0}$ are both zero or very small which means that there will not be any interesting display of CP-violation in colliders. Moreover, electroweak baryogenesis cannot take place in this case $[9$, $10]$.

- The contributions of the phases of $\mu$ and $a_{0}$ cancel each other. From the phenomenological point of view, this is the most interesting solution because it leaves room for a host of non-trivial CP-violating as well as CP-conserving phenomena to be discovered.

The third possibility has been extensively studied in the literature [11] and unfortunately it seems that cancelation scenario works only if the phase of $\mu$ is $\mathcal{O}\left(10^{-2}\right)$ or less which is too small to result in detectable CP-violating effects in colliders. This is due to two reasons: 1) The severe upper bounds on $d_{H g}$ and $d_{n}$ have to be simultaneously satisfied that is while there are only two CP-violating phases. It seems natural that in the parameter range that the contributions of $\theta_{a_{0}}$ and $\theta_{\mu}$ to $d_{e}$ cancel each other, their contribution to $d_{H g}$ add up and vice versa. 2) In the large $\tan \beta$ regime (which is favored by the LEPII data [3] as well as the $\mathrm{SO}(10)$ model [12]), the contribution of $\theta_{\mu}$ to the EDMs of the electron as well as the down quark is enhanced such that it cannot be canceled by the effect of the phase of $a_{0}$, unless the phase of $\mu$ itself is small. This means that, for large $\tan \beta$ regime, even if we relax the condition of universality of the $A$-terms at the GUT scale (taking $A_{e}$, 
$A_{u}$ and $A_{d}$, at the low energy scale, arbitrary), cancelation condition can be hardly satisfied [13].

In this paper, we relax some of the universality conditions and find that for a range of parameters, which from phenomenological point of view is interesting, the cancelation scenario is revived even for intermediate values of $\tan \beta(\tan \beta \simeq 10)$. This basically happens when the masses of sfermions are low while the values of $M_{2}$ (Wino mass), $A_{e}$ and $A_{d}$ are large. Putting it in another way, the suppression scenario discussed here engages two of the above conditions: cancelation as well as having large mass parameters. However, we only take some of the parameters large; i.e., there is a small hierarchy of order of 10 among the supersymmetric parameters at the electroweak scale. Such a hierarchy is not by any means peculiar to this model. Even in the context of the mSUGRA model, at the low scale the colored particles are expected to be (5-10) times heavier than those which are singlets of SU(3).

In section 2, we describe the model and discuss that having $A_{e}$ and $A_{d}$ at the $\mathrm{TeV}$ scale can be consistent with the bounds from the Color and Charge Breaking (CCB) vacua considerations. In section 3, we study the behavior of EDMs by varying different parameters and discuss the robustness of our results. In section 4, we study the possibility of cancelation in the parameter range favored by resonant electroweak baryogenesis. In section 5 , we discuss if the cancelation opens the possibility of large enough phases to cause sizeable CP-violating effects in colliders. Conclusions are summarized in section 6 .

\section{The model}

In this paper, we consider the Minimal Supersymmetric Standard Model with superpotential

$$
W_{M S S M}=Y_{u} \widehat{u^{c}} \widehat{Q} \cdot \widehat{H_{u}}-Y_{d} \widehat{d^{c}} \widehat{Q} \cdot \widehat{H_{d}}-Y_{e} \widehat{e^{c}} \widehat{L} \cdot \widehat{H_{d}}-\mu \widehat{H_{u}} \cdot \widehat{H_{d}}
$$


where, $\widehat{L}, \widehat{Q}, \widehat{H_{u}}, \widehat{H_{d}}$ are doublets of chiral superfields associated respectively with left-handed leptons, left-handed quarks and the two Higgs doublets of the MSSM. In the above formula, $\widehat{u^{c}}, \widehat{d^{c}}$ and $\widehat{e^{c}}$ are the chiral superfields associated with the corresponding right-handed fields. The soft supersymmetry breaking part of Lagrangian, at the electroweak scale, is taken to have the form

$$
\begin{aligned}
\mathrm{E}_{\mathrm{soft}}^{\mathrm{MSSM}} & =-1 / 2\left(M_{3} \widetilde{g} \widetilde{g}+M_{2} \widetilde{W} \widetilde{W}+M_{1} \widetilde{B} \widetilde{B}+\text { H.c. }\right) \\
& -\left(A_{u i} Y_{u i i} \widetilde{u_{i}}{ }^{c} \widetilde{Q_{i}} \cdot H_{u}-A_{d i} Y_{d i i} \widetilde{d_{i}^{c}} \widetilde{Q_{i}} \cdot H_{d}-A_{e i} Y_{e i i} \widetilde{e_{i}^{c}} \widetilde{L_{i}} \cdot H_{u}+\text { H.c. }\right) \\
& -{\widetilde{Q_{i}}}^{\dagger} m_{\tilde{Q_{i i}}}^{2} \widetilde{Q_{i}}-{\widetilde{L_{i}}}^{\dagger} m_{\tilde{L} i i}^{2} \widetilde{L_{i}}-\widetilde{\left(u_{i}^{c}\right)^{\dagger}} m_{\tilde{u} i i}^{2} \widetilde{u_{i}^{c}}-\widetilde{\left(d_{i}^{c}\right)^{\dagger}} m_{\tilde{d} i i}^{2} \widetilde{d_{i}^{c}}-\widetilde{e_{i}^{c}} m_{\tilde{e} i i}^{2} \widetilde{e_{i}^{c}} \\
& -m_{H_{u}}^{2} H_{u}^{\dagger} H_{u}-m_{H_{d}}^{2} H_{d}^{\dagger} H_{d}-\left(B_{H} H_{u} \cdot H_{d}+\text { H.c. }\right),
\end{aligned}
$$

where the " $i$ " indices determine the flavor. We have relaxed the universality assumption ( $i . \quad e$., in general, $m_{\tilde{\mu}}^{2} \neq m_{\tilde{e}}^{2} \neq m_{H_{u}}^{2} \neq$ etc); however, we have taken a flavor conserving soft potential (i.e., there is no off-diagonal terms in the flavor basis). The latter assumptions is motivated by observation [14]. We have defined the $A$-parameters factoring out the corresponding Yukawa couplings. Notice that since we do not assume any universality, from the beginning we concentrate on the potential at the electroweak scale rather than some high GUT scale. Hermiticity of the Lagrangian implies that $m_{H_{u}}^{2}$, $m_{H_{d}}^{2}$ and the sfermion masses are real. The rest of parameters in Eq. 3 can in general be complex. By rephasing the fields we can make $B_{H}$ and $M_{2}$ real but, in this basis the phases of $\mu$ - and $A$-parameters as well as $M_{3}$ and $M_{1}$ cannot be rotated away and should be considered as new sources of CP-violation.

In Ref [15], the possibility of cancelation between contributions of the phases of $A_{e}, A_{d}, A_{u}, M_{1}, M_{3}$ and $\mu$ has been studied for $\left|A_{i}\right|<1 \mathrm{TeV}$ and $\tan \beta<10$. As expected, they have found that increasing $\tan \beta$, the range of $\theta_{\mu}$ for which cancelation is possible shrinks. In this paper, we focus on $\left|A_{s}\right|,\left|A_{d}\right|>1 \mathrm{TeV}$ and show that, for large values of $\left|A_{i}\right|$, cancelation scenario 
is revived even for the intermediate values of $\tan \beta$.

One can reconstruct models for which deviation from universality is partial. For example, it is shown [16] that in the context of type I seesaw mechanism embedded in the MSSM, the neutrino B-term radiatively induces different corrections to $A_{u}$ and $A_{e}$ (lifting their universality) however, in this model, the universality of gaugino masses (at the GUT scale) is maintained. Considering such possibilities, we first perform an analysis setting the phases of gaugino masses equal to zero and then allow the phases of $M_{1}$ and $M_{3}$ to be nonzero. Of course, allowing more phases to be nonzero, the likelihood of cancelation increases.

For large values of $A$-terms, one of course has to check the CCB bounds. In the following, we discuss that by relaxing the condition of universality at the GUT scale, we can have values of $A_{e}$ and $A_{d}$ as large as a few TeV while keeping the sfermion masses below $\mathrm{TeV}$ without encountering Color or Charge Breaking (CCB) vacua. In the end, we suggest a way to test the preassumption that goes into this possibility.

As it is well-known, at the tree level, the conditions for the electroweak symmetry breaking are

$$
|\mu|^{2}+m_{H_{d}}^{2}=B_{H} \tan \beta-\frac{m_{Z}^{2}}{2} \cos 2 \beta
$$

and

$$
|\mu|^{2}+m_{H_{u}}^{2}=B_{H} \cot \beta+\frac{m_{Z}^{2}}{2} \cos 2 \beta .
$$

For large $\tan \beta$, we can neglect $B_{H} \cot \beta$ and write

$$
\begin{aligned}
& m_{H_{u}}^{2} \simeq-|\mu|^{2}+\frac{m_{Z}^{2}}{2} \cos 2 \beta \\
& m_{H_{d}}^{2} \simeq B_{H} \tan \beta+m_{H_{u}}^{2}-m_{Z}^{2} \cos 2 \beta
\end{aligned}
$$

so we expect $m_{H_{u}}^{2}$ to be negative. In the mSUGRA, the values of $m_{H_{u}}^{2}, m_{H_{d}}^{2}$ as well as the sfermion and gaugino masses are all determined by two parameters 
$m_{0}$ and $m_{1 / 2}$. This means that to have a low spectrum, $B_{H} \tan \beta$ should be small, too. For large values of $\tan \beta$, this means that $B_{H}$ should be much smaller than other supersymmetric parameters which sounds unnatural (see however [17]). Now if we relax the unification of the masses at high energies and take $B_{H}$ to be of order of $\left|m_{H_{u}}^{2}\right|$, we find that $m_{H_{d}}^{2}$ can be positive and large.

For positive $m_{H_{d}}^{2}$ as discussed in [18] to guarantee that no CCB occurs, it is sufficient to have ${ }^{1}$

$$
A_{e}^{2}<3\left(m_{H_{d}}^{2}+m_{\tilde{e}_{L}}^{2}+m_{\tilde{e}_{R}}^{2}\right)
$$

and

$$
A_{d}^{2}<3\left(m_{H_{d}}^{2}+m_{\tilde{d}_{L}}^{2}+m_{\tilde{d}_{R}}^{2}\right)
$$

The larger $m_{H_{d}}^{2}$ and $B_{H} / \cos \beta$, the less stringent the upper bounds on $A_{e}$ and $A_{d}$. Remembering that the masses of CP-odd Higgs field $\left(A^{0}\right)$, the heavier CP-even Higgs $\left(H^{0}\right)$ and charged Higgses $\left(H^{ \pm}\right)$are given by $\left(2 B_{H} / \sin 2 \beta\right)^{1 / 2}$, the assumption of large $m_{H_{d}}^{2}$ can put into test at the LHC [19]. Finally, since we are assuming that off-diagonal elements of the $A$-terms are absent, we do not need to be concerned about the region unbounded from below [20].

\section{$3 \quad$ Numerical results}

In this section, we study the electric dipole moments of the electron, mercury and neutron to find ranges of phases for which cancelation is possible. We first discuss the constraints on the input parameters from various observations with special emphasis on the uncertainty in the input parameters and theoretical formulae. We then discuss how, by varying the input variables, the overall behavior of the bounds changes. We then analyze the possibility

\footnotetext{
${ }^{1}$ The bounds $(6,7)$ are based on tree-level analysis. However, [20] shows that loop effects do not affect these results.
} 
of cancelation in the following two cases that are phenomenologically interesting: 1) close to the benchmark SPS1a' [21] (三B" [22]); 2) the range $\mu \simeq M_{1}$ where the Higgsino-Bino mixing is large. The latter case is of interest because in this regime, neutralino annihilation is relatively large, yielding the desirable dark matter density.

We assign various values in the relevant range to $\mathrm{CP}$-conserving parameters of the model $(\tan \beta$, the $\mu$ parameters, the $A$-parameters, the selectron masses, the masses of superpartners of light quarks and gaugino masses). As it is well-known, the condition for electroweak symmetry breaking determines the values of $\mu$ in terms of $m_{H_{d}}^{2}, m_{H_{u}}^{2}$ and $\tan \beta$. In this paper, as we discussed earlier we do not make a priori any assumption on the values of $m_{H_{u}}^{2}$ and $m_{H_{d}}^{2}$ so we are free to assign any value to $|\mu|$. In this regard, our model resembles the Non-Universal Higgs Mass (NUHM) model which has recently received attention in the literature [23].

One of the triumphs of the MSSM is providing a natural candidate for the dark matter; i.e. the lightest neutralino $\left(\tilde{\chi}_{1}^{0}\right)$. In order for the relic density of neutralinos to satisfy the precise results of WMAP, the parameter space of the constrained MSSM is reduced to narrow strips in the $m_{0}-m_{1 / 2}$ plane for given values of $\tan \beta$ and $A_{0}$ [24]. The contribution of neutralino to Dark matter energy density is determined by annihilation rate after the temperature drops below its mass. In the present model, it is possible to tune the (co)annihilation rate of neutralino to a value that explains the data: For the parameter range that we have chosen $\tilde{\chi}_{1}^{0}$ has a large $\tilde{B}^{0}$ component which means it has a large annihilation cross section with $\tilde{\tau}_{R}$. If the mass of $\tilde{\tau}_{R}$ is close to that of $\tilde{\chi}_{1}^{0}$, for temperatures slightly below their mass, their density will be comparable. This means the coannihilation rate of neutralinos with $\tilde{\tau}_{R}$ can be large enough to bring the contribution of neutralinos to the dark matter density to the desired values. Notice that in this scenario the dark matter density is not sensitive to the value of $M_{2}$ (Wino mass) because (i) 
the $\tilde{W}^{0}$ component of $\tilde{\chi}_{1}^{0}$ is small; (ii) $\tilde{\tau}_{R}$ does not couple to $\tilde{W}^{0}$. So, varying the values of $M_{2}$ (as in Fig. 1), will not considerably affect the neutralino density.

Another major constraint on the MSSM parameters comes from the radiative correction to $\operatorname{Br}(b \rightarrow s \gamma)$ (see e.g., [25]). The leading 1-loop SUSY diagrams involve loops with a charged Higgs and a top quark and loops with a chargino and a squark. Since in the present analysis the deviation from the cMSSM spectrum is in the direction of increasing $M_{2}$ and $B_{H}$ (and hence $m_{\tilde{\chi}^{+}}$and $\left.m_{H^{+}}\right)$the SUSY correction to $b \rightarrow s \gamma$ is further suppressed and as a result the bounds from $b \rightarrow s \gamma$ cannot be significant.

To calculate the EDMs and CEDMs of the elementary particles, we use the formalism developed in [26]. In the literature, there are various different formulae for the EDM of mercury:

- according to $[27]$

$$
d_{H g}=-\left(\tilde{d}_{d}-\tilde{d}_{u}-0.012 \tilde{d}_{s}\right) \times 3.2 \cdot 10^{-2} e,
$$

where $\tilde{d}_{d}, \tilde{d}_{u}$ and $\tilde{d}_{s}$ are respectively the chromoelectric dipole moments of the $d, u$ and $s$ quarks.

- that is while according to [28]

$$
d_{H g}=8.7 \times 10^{-3} \times e\left(\tilde{d}_{d}-\tilde{d}_{u}-0.0051 \tilde{d}_{s}\right)
$$

In this paper, we study the both formulae and discuss the effects of the theoretical uncertainty.

In the literature, to calculate the EDM of neutron various theoretical approaches have been taken which give different and even conflicting results. For example, the QCD sum rules yield [29]

$$
d_{n}=(1 \pm 0.5) \frac{|\langle\bar{q} q\rangle|}{(225 \mathrm{MeV})^{3}} \times\left[0.55 e\left(\tilde{d}_{d}+0.5 \tilde{d}_{u}\right)+0.7\left(d_{d}-0.25 d_{u}\right)\right]
$$


while SU(3) chiral Lagrangian technique [30] gives

$$
d_{n}=\left(1.6 \times \tilde{d}_{u}+1.3 \times \tilde{d}_{d}+0.26 \times \tilde{d}_{s}\right) \text { e cm } .
$$

Notice that since we expect $\tilde{d}_{s} / \tilde{d}_{d} \sim m_{s} / m_{d} \simeq 19$ [31], in the latter formula the dominant contribution is that of the strange quark whose effect is completely neglected in the former formula. On the other hand, in the latter formula the effects of EDM of quarks are neglected and only chromoelectric dipole moments are considered. Because of these theoretical uncertainties, we do not put much emphasis on the bounds from neutron EDM. In our calculation, we will be using the formulation in [30].

Figs $(1,2)$ display the maximum values of $\theta_{\mu}$ for which cancelation between the contributions of the phases of $A_{e}$ and $\mu$ is possible. Since the masses of selectrons and sneutrino are taken to be relatively small $(<1 \mathrm{TeV})$, the dominant effects are given by one-loop chargino and neutralino exchanges [26] and the effect of two-loop diagrams can be neglected [32].

Drawing Fig. 1, the phases of gauginos are set equal to zero. From Fig. 1 we conclude that with growing $M_{2}$, cancelation for larger values of $\theta_{\mu}$ becomes possible. This is because the dominant contribution to $d_{e}$ comes from the chargino exchange which depends only on the phase of $\mu$. That is while only the subdominant effect (the neutralino exchange diagram) depends on the phase of $A_{e}$. Increasing $M_{2}$, the effect of chargino exchange is suppressed and in turn cancelation between two effects will be possible for higher values of $\theta_{\mu}$. As expected, increasing the value of $\left|A_{e}\right|$, the range of values of $\theta_{\mu}$ for which cancelation is possible is enlarged. This can be observed by comparing curves (a1) with (a2); (a3) with (a4); and (b1) with (b2). Increasing tan $\beta$ from 10 to 20 , the maximum values for which cancelation is possible is considerably reduced [see curves (b1) and (b2)]. Comparing curves (a1) and (a3) with (c1), (c2) and (c3), we observe that by increasing the masses of the supersymmetric particles the maximum $\theta_{\mu}$ for which cancelation is possible is reduced. This 
rather counter-intuitive behavior is also observed by [15] for lower values of $\tan \beta$ and $\left|A_{e}\right|$. However, as expected for larger values of sfermion masses the degree of fine tuning of the phases necessary for cancelation is smaller; i.e., for larger sfermion masses, the value of $d_{e}^{\text {exp }} / d_{e}\left(\theta_{\mu}, \theta_{A_{e}}=\right.$ arbitrary) is smaller. Finally comparing curves (c1), (c2) and (c3) with each other and contrasting (a1) and (a2) versus (a3) and (a4), we observe that the results are robust against varying the values of $|\mu|$.

Fig. 2 shows maximum and minimum values of $\theta_{\mu}$ for which cancelation among the contributions of the phases of $M_{1}, A_{e}$ and $\mu$ is possible. Each curve corresponds to a different value of $\theta_{M_{1}}$ as indicated. For input parameters, we have chosen the spectrum of the SPS1a' benchmark which is compatible with all the present bounds and will be investigated by the LHC [21]. As far as the EDM bounds are concerned, this benchmark is a typical of points close to it. We confirmed the robustness of these results by varying the input parameters around this point. When $\theta_{\mu}$ and $\theta_{M_{1}}$ have opposite signs, cancelation becomes possible for larger values of $\left|\theta_{\mu}\right|$ than in the case that the relative sign is positive. In the latter case, even values of $\left|\theta_{\mu}\right|$ of order of one are compatible with the bounds on $d_{e}$. Notice that the results are symmetric under $\theta_{\mu} \rightarrow-\theta_{\mu}$ and $\theta_{M_{1}} \rightarrow-\theta_{M_{1}}$. If future searches put stronger bounds on $d_{e}$, our results will still be valid but a greater degree of fine tuning for successful cancelation will be required.

Let us now discuss the possibility of cancelation between different contributions to $d_{H g}$. This possibility has been studied in Fig. (3). Since, at the electroweak scale, $m_{H_{u}}^{2}$ is negative (electroweak symmetry breaking implies $m_{H_{u}}^{2} \simeq-|\mu|^{2}$ ), the CCB bounds severely restrict the value of $\left|A_{u}\right|$. That is why we have taken $\left|A_{u}\right|=300 \mathrm{GeV} \ll\left|A_{d}\right|,\left|A_{s}\right|$. For a given set of phases, the contribution of $\tilde{d}_{u}$ to $d_{H g}$ is subdominant. This is expected because (i) $\left|A_{u}\right| \ll\left|A_{d}\right|,\left|A_{s}\right|$; ii) the dominant contribution from $\theta_{\mu}$ to $\tilde{d}_{u}$ is suppressed by $\cot \beta$; that is while the corresponding contribution to $\tilde{d}_{d}$ and $\tilde{d}_{s}$ is en- 
hanced by $\tan \beta$. In general, we expect the uncertainty in the values of $m_{d}$ and $m_{s}$ to dramatically affect the values of the calculated EDMs. However, since the ratio $m_{s} / m_{d}$ is known to a rather high accuracy [31] the region of parameter space in which cancelation occurs is not considerably affected by the uncertainty in knowledge of $m_{d}$. Nevertheless, the theoretical uncertainty in calculating $d_{H g}[$ e.g., Eq. (9) vs. Eq. (8)] can change the conditions for cancelation to some extent.

Fig. 3 displays the maximum values of $\theta_{\mu}$ for which cancelation among the contributions of the phases of $M_{3}, \mu, A_{d}, A_{s}$ and $A_{u}$ to $d_{H g}$ is possible. Each curve corresponds to a different value of $\theta_{M_{3}}$. The same as in Fig. 2, we have set the input parameters close to the benchmark SPS1a' [21]. For a given value of $\theta_{M_{3}}$, using the formula given in [28] [see Eq. (9)] the bound on $d_{H g}$ appears more restrictive than the formula given by the QCD sum rule [27] [see Eq. (8)]. This can be observed from Fig. 3: the thin discrete points lie above the thick points connected by lines. This is expected because when the contribution of $\tilde{d}_{s}$ is taken to be large, the phase of $A_{s}$ can play a greater role in canceling the other effects. From Fig. 3, we conclude that the cancelation scenario is open for a wider range of $\theta_{\mu}$ if the relative sign of $\theta_{M_{3}}$ and $\theta_{\mu}$ is positive. This is because of the partial cancelation that occurs between their respective contributions in this case. When both $\theta_{M_{3}}$ and $\theta_{\mu}$ are positive, even phases close to $\pi / 2$ are compatible with the bound on $d_{H g}$.

Remember that we have allowed the $A$-parameters of the electron and quarks to be different from each other, so the degrees of freedom are enough to simultaneously fulfill the cancelation conditions for $d_{e}$ and $d_{H g}$ with nonvanishing solutions for phases. However one should check if there is an overlap between the range of $\theta_{\mu}$ and $\theta_{M_{1}}$ for which $d_{e}$ and $d_{H g}$ can vanish because of cancelation. Relaxing the assumption of universality, this comparison is rather non-trivial because $d_{H g}$ and $d_{e}$ are sensitive to different sets of input parameters. Since the mass spectrum for both figures 2 and 3 correspond 
to the same benchmark, it makes sense to compare them. To make such a comparison, we should notice that 1) $d_{e}$ does not depend on $\theta_{M_{3}}$;2) sensitivity of $d_{H g}$ to $\theta_{M_{1}}$ is low. Comparison shows that we can simultaneously satisfy both bounds for $\left|\theta_{\mu}\right| \sim \mathcal{O}(1)$. Figs. 4 also show the range of $\left(\theta_{\mu}, \theta_{M_{1}}\right)$ [or $\left.\theta_{\mu}, \theta_{M_{3}}\right]$ for which cancelation is possible. To draw these figures, we have taken the spectrum of SPS1a' as input for masses but we have taken $\left|A_{e}\right|=$ $2000 \mathrm{GeV},\left|A_{u}\right|=300 \mathrm{GeV}$ and $\left|A_{s}\right|=\left|A_{d}\right|=3000 \mathrm{GeV}$. Fig (4-a) shows the range of $\theta_{\mu}$ and $\theta_{M_{1}}$ for which cancelation between contributions of phases of $\mu, M_{1}$ and $A_{e}$ to $d_{e}$ is possible. Notice that even $\theta_{\mu}=\pi / 2$ can be compatible with the upper bound on $d_{e}$. In the area shadowed in Fig. (4-b), cancelation between contributions of the phases of $\mu, M_{3}, A_{u}, A_{d}$ and $A_{s}$ to $d_{n}$ and $d_{H g}$ can suppress $d_{n}$ and $d_{H g}$ to values below the strong bounds on them. To draw Fig. (4-b), we have set $\theta_{M_{1}}=0$. Fig. (4-c) is similar to Fig. (4-b) except that in the case of Fig. (4-c), $\theta_{M_{3}}$ instead of $\theta_{M_{1}}$ is set equal to zero. Fig. (4-d) displays the range of $\theta_{\mu}$ and $\theta_{M_{1}}$ for which cancelation between different contributions to $d_{n}$ and $d_{H g}$ is possible. Drawing Fig (4-d) the phases $\theta_{\mu}$, $\theta_{M_{1}}, \theta_{M_{3}}, \theta_{A_{d}}, \theta_{A_{u}}$ and $\theta_{A_{s}}$ are all allowed to vary in order to satisfy the bounds on $d_{n}$ and $d_{H g}$. As expected, Figs. (4-c) and (4-d) look similar but Fig. (4-d) covers a larger range because in this case there is one more degree of freedom to satisfy the bounds. To simultaneously satisfy all the bounds, the values of $\theta_{\mu}$ and $\theta_{M_{1}}$ should be in the overlap of Figs (4-a) and (4-d). Contrasting these two figures we find out that values of $\theta_{\mu}$ and $\theta_{M_{1}}$ of order of 1 are possible. It is interesting that the maximal CP-violating case $\theta_{\mu}= \pm \frac{\pi}{2}$ which is compatible with $d_{e}$ is excluded by the bounds on $d_{H g}$ and $d_{n}$ and on the other hand, $\theta_{M_{1}}= \pm \frac{\pi}{2}$ which is compatible with the $d_{n}$ and $d_{H g}$ bounds is ruled out by bound on $d_{e}$. One should be aware that in this range of parameters the bound from $d_{n}$ is more restrictive than the bound from $d_{H g}$. If we redraw the Figs. (4-b) to (4-d) overlooking the bound on $d_{H g}$, we find that results do not considerably change. This shows that in order to 
derive conclusive results from EDMs, solving the theoretical uncertainties in calculation of $d_{n}$ is imperative.

From cosmological point of view, the region $\mu \simeq M_{1}$ is specially interesting because in this region, the Higgsino Bino mixing is sizeable yielding relatively large $\tilde{\chi}_{1}^{0} \tilde{\chi}_{1}^{0}$ annihilation rate in accord with the dark matter density measurements. Recently it has been shown [33] that varying the values of the CP-violating phases, the range of parameters compatible with the measured dark matter density will be enlarged. Fig. 5 tries to find out if large $\mathrm{CP}$-violating phases, for sub-TeV selectron masses, are allowed. From these figures, we find out that although $\theta_{M_{1}}$ can be $\mathcal{O}(\pi / 2), \theta_{\mu}$ cannot exceed $0.1 \pi$ even if $A_{e}=4000 \mathrm{GeV}$. We have checked the robustness of this result by varying the parameters along $\mu \simeq M_{1}$ and the result seems generic.

\section{$4 \quad$ EDM bounds and electroweak baryogenesis}

Arguably the most important manifestation of $\mathrm{CP}$-violation is its role in the creation of the baryon asymmetry of the Universe. In the context of MSSM, all three Sakharov's famous conditions can be fulfilled and, in principle, through a mechanism known as electroweak baryogenesis, the baryon asymmetry of the Universe can be explained. To have strong first order electroweak phase transition necessary for the creation of the baryon asymmetry of the universe, one of the top squarks should be lighter than the top quark. If we demand the lightest neutralino to be the lightest supersymmetric particle, this in turn implies $m_{\tilde{\chi}_{1}^{0}}<m_{t}$. The other requirement for having successful electroweak baryogenesis is of course having large enough CP-violating phases. However, in [10] it is shown that even for values of $\sin \theta_{\mu}$ as low as $10^{-2}$ successful electroweak baryogenesis can be a possibility provided that we are at the resonance region $[34,35]$ (i. e., $|\mu| \simeq\left|M_{1}\right|$ or $|\mu| \simeq\left|M_{2}\right|$ ). Moreover to have a successful electroweak baryogenesis the mass of the CP- 
odd Higgs boson, $m_{A^{0}}$, should be relatively low $\left(m_{A^{0}} \ll \mathrm{TeV}\right)$. Notice that if the masses of selectron and sneutrino are below the TeV scale, even values of $\sin \theta_{\mu}$ as low as $10^{-2}$ will not be compatible with the bounds on the electric dipole moment of electron, unless the cancelation scenario is at work. Suppose future experiments (the LHC and ILC) confirm supersymmetry and find out that $m_{\tilde{\chi}_{1}^{0}}<m_{\tilde{t}_{R}}<m_{t}$ and discover a relatively light $A^{0}$. These conditions are tantalizingly close to the requirement for a successful electroweak baryogenesis. Now, suppose that the masses of selectrons turn out to be at the scale of few hundred GeV. Does this mean that the electroweak baryogenesis is ruled out? Figs $(6,7)$ try to address this question by studying the possibility of cancelation between different contributions to $d_{e}$. As we discussed in the previous section, since we have set the masses of sfermions below the TeV scale, the one-loop effects dominate over the two-loop effects. Taking the two-loop effects into account only slightly shifts the cancelation point.

To draw Fig 6, we have set $\tan \beta=10, m_{\tilde{e}_{L}}=392 \mathrm{GeV}, m_{\tilde{e}_{R}}=218 \mathrm{GeV}$, $m_{\tilde{\nu}_{L}}=385 \mathrm{GeV}$, and $M_{2}=415 \mathrm{GeV}$. Moreover we have set $\left|M_{1}\right|=|\mu|=200$ $\mathrm{GeV}$ which means we are in the neutralino-driven resonant electroweak baryogenesis regime [10]. For this choice of parameters the lightest neutralino is indeed lighter than the top quark. We have set $A_{e}=700 \mathrm{GeV}$ which is smaller than $\left[3\left(m_{\tilde{e}_{L}}^{2}+m_{\tilde{e}_{R}}^{2}\right)\right]^{1 / 2}$ thus, as long as $m_{H_{d}}^{2}$ is positive [18], no CCB will take place (see Eq. 6). Positiveness of $m_{H_{d}}^{2}$ sets a lower bound on $b \tan \beta \simeq m_{A^{0}}^{2}$ [see Eq. (4)] which for our choice of parameters is $190 \mathrm{GeV}$. Thus, for these parameters $A^{0}$ (the CP-odd Higgs boson) can still be sufficiently light. Increasing $A_{e}$ the cancelation can of course become possible for larger values of $\theta_{\mu}$ but the lower bound on $m_{A^{0}}$ will also be stronger and on the other hand, for heavier $m_{A^{0}}$ the produced baryon asymmetry is suppressed. As shown in [10], the neutralino-driven resonant baryogenesis is only marginally compatible with the indirect searches of dark matter so this choice of parameters 
in near future will be tested not only by collider data but also by further indirect dark matter searches.

From Fig. 6, we observe that for universal gaugino masses $\left[\theta_{M_{1}}=\theta_{M_{2}}=\right.$ $0]$, cancelation can take place even for values of $\left|\sin \theta_{\mu}\right|$ up to 0.06 which according to [10] can easily yield the baryon-antibaryon asymmetry compatible with the WMAP results. This confirms the results of [34]. In the neutralino-driven electroweak baryogenesis regime, the combination of the phases which determines baryogenesis is $\theta_{\mu}+\theta_{M_{1}}[36]$. Notice that $\theta_{\mu}+\theta_{M_{1}}$ is a rephasing invariant quantity. Fig 6 shows that, relaxing the assumption of the universality of the gaugino masses $\left[\theta_{M_{1}} \neq \theta_{M_{2}}=0\right]$, cancelation makes $\left|\sin \left(\theta_{\mu}+\theta_{M_{1}}\right)\right| \sim 1$ compatible with the bounds on $d_{e}$.

Now let us discuss fine tuning required for such cancelation. If the phases of $\mu$ and $M_{1}$ are at the region where cancelation can take place, the generic value of $d_{e}$ is already around $10^{-26}$ e $\mathrm{cm}$ so to reduce the value of $d_{e}$ down to below the upper bound on it (see Eq. 1), a cancelation of $10 \%$ will be enough which means the fine tuning of the phases is not a problem. It can be shown that for this range of $\theta_{\mu}$ and $\theta_{M_{1}}$, the different contributions from phases of $M_{3}, M_{1}, \mu, A_{d}$ and $A_{s}$ to $d_{n}$ and $d_{H g}$ can also cancel each other to satisfy the experimental upper bounds. The degree of fine-tuning necessary for suppressing the values of $d_{n}$ and $d_{H g}$ down to the values below the upper bounds on them depends on the scale of $M_{3}$ and squark masses as well as $A$-terms. We have checked the degree of fine tuning needed for effective cancelation setting $M_{3} \simeq 1400 \mathrm{GeV}$ and $m_{\tilde{d}}=1200 \mathrm{GeV}$ (these are the values corresponding to the prediction of cMSSM if the values of $M_{1}$ and selectron are as above). We have set $A_{s}=A_{d}=900 \mathrm{GeV}$ which are smaller than $\sqrt{3\left(m_{\tilde{d}_{L}}^{2}+m_{\tilde{d}_{R}}^{2}\right)}$. For the phases in the shadowed region shown in Fig 6 cancelation required in order to suppress $d_{H g}$ below the upper bound on it is not stronger than $\sim 5 \%(\sim 20 \%)$ if we take the formula in [27] $([28])$. We have also found that cancelation required in order to suppress $d_{n}$ below 
its upper bound is not stronger than $\sim 3 \%(\sim 15 \%)$ if we take the formula in [30] $([29])$. In near future, the experiments are going to become sensitive to even smaller values of $d_{n}, d_{H g}$ and $d_{e}$. Moreover, there are proposals to probe EDM of deuteron down to $(1-3) \times 10^{-27}$ e $\mathrm{cm}$ [37]. If one or more of these experiments detect a nonzero electric dipole moment, it will be a strong hint in favor of the electroweak baryogenesis. On the other hand, if they all report null results, we still cannot rule out the cancelation scenario even though a new piece of information (the bound on $d_{D}$ ) is added. However a greater degree of fine tuning would be necessary for the cancelation.

Fig. 7 explores the possibility of cancelation scenario and having large CP-violating phases in the chargino-driven resonant electroweak baryogenesis regime $\left(|\mu| \simeq M_{2}\right)$. The above discussion holds in this case, too, with the difference that for the chargino-driven electroweak baryogenesis the combination of phases that relevant for baryogenesis is $\theta_{\mu}+\theta_{M_{2}}$. According to this figure $\sin \left(\theta_{\mu}+\theta_{M_{1}}\right)$ can reach 0.09 which may be enough for a successful baryogenesis [10]. In case of Fig. 7, since $|\mu|$ is larger, the lower bound on the CP-odd Higgs boson will be stronger: $m_{A^{0}}>335 \mathrm{GeV}$. Unlike the case of neutralino-driven electroweak baryogenesis, the chargino-driven electroweak baryogenesis is not sensitive to the indirect dark matter searches.

\section{Implication of cancelation scenario for CP- violation searches in the colliders}

The CP-violating phases can manifest themselves as both CP-even and CPodd quantities in the LHC [7, 38] and International Linear Collider, ILC $[39,40,41]$. Due to high precision and capability of polarizing the initial beams, the ILC will have a greater chance to observe CP-violation in the production and decay of sparticles. In $[40,41]$, it is shown that even small values of CP-violating phases can result in an asymmetry between $e^{+} e^{-} \rightarrow$ 
$\tilde{\chi}_{1}^{0} \tilde{\tau}_{1}^{+} \tau^{-}$and $e^{+} e^{-} \rightarrow \tilde{\chi}_{1}^{0} \tilde{\tau}_{1}^{-} \tau^{+}$. Following [40], let us define

$$
A_{C P} \equiv \frac{P_{2}-\bar{P}_{2}}{2}
$$

In the above definition, $P_{2}$ is the polarization of $\tau$ which is produced in the subsequent processes $e^{+} e^{-} \rightarrow \tilde{\chi}_{1}^{0} \tilde{\chi}_{i}^{0}$ and $\tilde{\chi}_{i}^{0} \rightarrow \tau^{-} \tilde{\tau}^{+}$. The polarization vector is defined as

$$
\vec{P} \equiv \frac{\operatorname{Tr}[\rho \vec{\sigma}]}{\operatorname{Tr}[\rho]}
$$

where $\rho$ is the spin density of $\tau$ and direction 2 is taken to be perpendicular to the plane defined by the momenta of the $\tau$ and the initial electron. Curves in Fig. 8, which are borrowed from Fig. 2.12.b of [40], show different contour lines corresponding to various values of $A_{C P}$. The input data for the curves are $\theta_{A_{\tau}}=0, A_{\tau}=250 \mathrm{GeV}$ and $\left(P_{e^{-}}, P_{e^{+}}\right)=(-0.8,0.6)$. The rest of the input parameters are given in the caption of Fig. 8. Notice that the input parameters satisfy the relations that we would have expected in the cMSSM. It is remarkable that $A_{C P}= \pm 45 \%$ can be possible for values of $\theta_{\mu}$ as small as $\pm 0.1 \pi$ and $\theta_{M_{1}}= \pm 1 / 6 \pi$ or for $\theta_{\mu}= \pm 0.06 \pi$ and $\theta_{M_{1}}= \pm \pi / 2$. The shadowed areas superimposed on the curves show the region for which the cancelation scenario can result in vanishing $d_{e}$. In order to check if in the same area vanishing $d_{n}$ and $d_{H g}$ is possible, we calculated the corresponding gluino and squark masses in the specific point in the cMSSM space chosen above and inserted them in the formulae for $d_{H g}$ and $d_{n}$. We found that for any given set of $\theta_{\mu}$ and $\theta_{M_{1}}$ total cancelation can simultaneously suppress the values of $d_{n}$ and $d_{H g}$. The overlap of curves with the shadowed area indicates that even for light sfermion masses, we still have a hope to observe CP-violating effects in the ILC provided that the systematic and statistical errors are under control.

Let us now discuss the fine tuning required for suppressing the EDMs below the upper bounds on them. Taking $\theta_{\mu}$ and $\theta_{M_{1}}$ in the shadowed area 
and assigning a general value between $-\pi$ and $\pi$ to $\theta_{A_{e}}$ we find that $d_{e}$ cannot exceed $10^{-26} \mathrm{e} \mathrm{cm}$. This means that the fine tuning required to suppress $d_{e}$ below the bound in Eq. 1 is not larger than 10\%. However, although simultaneous suppression of $d_{n}$ and $d_{H g}$ is possible for a wider range of phases, we have found that the required fine-tuning in this case is greater and is of order of $1 \%$.

\section{Conclusions}

In this paper, we have studied the possibility of satisfying the bounds on $d_{e}$, $d_{H g}$ and $d_{n}$ by cancelation scenario, relaxing the universality of parameters at the GUT scale. We have discussed that relaxing universality of the Higgs mass parameters $\left(m_{H_{d}}^{2}\right.$ and $\left.m_{H_{u}}^{2}\right)$ the color and charge breaking bounds on $A_{e}, A_{d}$ and $A_{s}$ will be less severe allowing $A$-terms of order of few $\mathrm{TeV}$. We have focused on the part of parameter space with intermediate values of $\tan \beta(\tan \beta \simeq 10)$ and $\mathrm{TeV}$ scale A-terms. This range has not been explored in the literature before. We have found that the bounds on $d_{e}$ and $d_{n}$, in this range, are complementary. We have argued that the uncertainty in evaluating $d_{n}$ in terms of the EDMs and CEDMs of the elementary particles causes large uncertainty in deriving bounds on the CP-violating phases. For the mass spectrum close to that of the SPS1a' benchmark (which will be soon explored at the LHC) $\left|\sin \theta_{\mu}\right|,\left|\sin \theta_{M_{1}}\right| \sim \mathcal{O}(1)$ is a possibility but requires high fine tuning $(0.1 \%)$.

We have also studied the possibility of cancelation for the region that electroweak baryogenesis is enhanced $\left(|\mu| \simeq\left|M_{1}\right|\right.$ and $\left.|\mu| \simeq\left|M_{2}\right|\right)$ and found that $\left|\sin \theta_{\mu}\right| \simeq 0.1$ and $\left|\sin \theta_{M_{1}}\right| \simeq 1$, even for the sub-TeV slepton masses, can be compatible with the EDM bounds. The main point is that relaxing the assumption of the universality of gaugino mass phases $\left(\theta_{M_{1}} \neq \theta_{M_{2}}\right)$ opens up the possibility of cancelation such that values of $\left|\sin \left[\theta_{\mu}+\theta_{M_{1}}\right]\right| \sim 1$ be- 
come compatible with the bounds on $d_{e}$. This opens new windows towards successful electroweak baryogenesis. Notice that in this range of parameters the fine-tuning required for successful cancelation is not too high.

We have explored the possibility of cancelation in the regime that the Higgino-Bino mixing is large. This part of the parameter space is of interest from neutralino relic density point of view. In the literature [33], it is shown that varying the $\mathrm{CP}$-violating phases in a large range broadens the parameter range compatible with the WMAP dark matter measurements. We find that in this regime, $\left|\sin \theta_{\mu}\right|$ cannot exceed 0.1 but $\left|\sin \theta_{M 1}\right|$ can reach 1 , even for relatively small sfermion masses.

We expect in the future, the running and planned experiments to probe smaller values of $d_{n}$ and $d_{H g}$ and $d_{e}$ [6]. Moreover the proposed experiments can probe $d_{D}$ with high sensitivity [37]. Even if all these experiments report null results, the range of parameters where cancelation is possible will not change but greater degree of fine tuning will be required.

\section{Acknowledgement}

We would like to thank D. Demir, J. Ellis, R. Godbole and M. Hashemi for the useful discussions. We are specially thankful to S. Profumo for pointing out a mistake in the first version of the manuscript. We appreciate O. Kittel for giving us the permission to use his plots. We are also grateful to $\mathrm{R}$. Asgari for teaching us how to use the software for drawing the plots. Y. F. is grateful to the CERN theory division for the hospitality during her stay at CERN when a part of this work was done. 


\section{References}

[1] W. Bernreuther and M. Suzuki, Rev. Mod. Phys. 63 (1991) 313 [Erratum-ibid. 64 (1992) 633]; Notice that a more recent analysis reports a substantially smaller value: M. J. Booth, arXiv:hep-ph/9301293.

[2] E. P. Shabalin, Sov. Phys. Usp. 26 (1983) 297 [Usp. Fiz. Nauk 139 (1983) 561]; M. B. Gavela et al., Phys. Lett. B 109 (1982) 215; I. I. Y. Bigi and N. G. Uraltsev, Sov. Phys. JETP 73 (1991) 198; I. B. Khriplovich and A. R. Zhitnitsky, Phys. Lett. B 109 (1982) 490.

[3] S. Eidelman et al. [Particle Data Group], Phys. Lett. B 592 (2004) 1.

[4] B. C. Regan, E. D. Commins, C. J. Schmidt and D. DeMille, Phys. Rev. Lett. 88 (2002) 071805.

[5] C. A. Baker et al., arXiv:hep-ex/0602020.

[6] Kawall et al., electron in AIP conf. Proc. 698 (2004) 192; S. K. Lamoreaux, arXiv:nucl-ex/0109014; Y. K. Semertzidis, Nucl. Phys. Proc. Suppl. 131 (2004) 244 [arXiv:hep-ex/0401016]; http://p25ext.lanl.gov/edm/edm.html.

[7] R. M. Godbole, Czech. J. Phys. 55 (2005) B221 [arXiv:hep-ph/0503088].

[8] T. Ibrahim and P. Nath, Phys. Lett. B 418 (1998) 98 [arXiv:hepph/9707409]; M. Brhlik, G. J. Good and G. L. Kane, Phys. Rev. D 59 (1999) 115004 [arXiv:hep-ph/9810457]; A. Bartl et al., Phys. Rev. D 60 (1999) 073003 [arXiv:hep-ph/9903402]; T. Falk, K. A. Olive, M. Pospelov and R. Roiban, Nucl. Phys. B 560 (1999) 3 [arXiv:hep$\mathrm{ph} / 9904393]$.

[9] V. A. Kuzmin, V. A. Rubakov and M. E. Shaposhnikov, Phys. Lett. B 155, 36 (1985). 
[10] V. Cirigliano, S. Profumo and M. J. Ramsey-Musolf, arXiv:hep$\mathrm{ph} / 0603246$.

[11] K. A. Olive, M. Pospelov, A. Ritz and Y. Santoso, Phys. Rev. D 72 (2005) 075001 [arXiv:hep-ph/0506106]; S. Abel, S. Khalil and O. Lebedev, Nucl. Phys. B 606 (2001) 151 [arXiv:hep-ph/0103320]; T. Falk et al., Nucl. Phys. B 560 (1999) 3 [arXiv:hep-ph/9904393]; A. Afanasev, C. E. Carlson and C. Wahlquist, Phys. Rev. D 61 (2000) 034014 [arXiv:hep-ph/9903493].

[12] H. Baer and J. Ferrandis, Phys. Rev. Lett. 87 (2001) 211803 [arXiv:hepph/0106352]; H. Baer, M. A. Diaz, J. Ferrandis and X. Tata, Phys. Rev. D 61 (2000) 111701 [arXiv:hep-ph/9907211]; H. Baer, M. Brhlik, M. A. Diaz, J. Ferrandis, P. Mercadante, P. Quintana and X. Tata, Phys. Rev. D 63 (2001) 015007 [arXiv:hep-ph/0005027]; T. Blazek, R. Dermisek and S. Raby, Phys. Rev. D 65 (2002) 115004 [arXiv:hepph/0201081]; T. Blazek, R. Dermisek and S. Raby, Phys. Rev. Lett. 88 (2002) 111804 [arXiv:hep-ph/0107097].

[13] T. Falk and K. A. Olive, Phys. Lett. B 439 (1998) 71 [arXiv:hepph/9806236]; T. Falk and K. A. Olive, Phys. Lett. B 375 (1996) 196 [arXiv:hep-ph/9602299].

[14] J. S. Hagelin, S. Kelley and T. Tanaka, Nucl. Phys. B 415 (1994) 293.

[15] V. D. Barger, T. Falk, T. Han, J. Jiang, T. Li and T. Plehn, Phys. Rev. D 64, 056007 (2001) [arXiv:hep-ph/0101106] and refrences therein.

[16] Y. Farzan, Phys. Rev. D 69 (2004) 073009 [arXiv:hep-ph/0310055]; Y. Farzan, JHEP 0502 (2005) 025 [arXiv:hep-ph/0411358]; Y. Farzan, arXiv:hep-ph/0505004. 
[17] H. Baer, J. Ferrandis and X. Tata, Phys. Lett. B 561 (2003) 145 [arXiv:hep-ph/0211418].

[18] J. F. Gunion et al., Nucl. Phys. B306 (1988) 1.

[19] M. Baarmand, M. Hashemi, A. Nikitenko, in press.

[20] J. A. Casas, arXiv:hep-ph/9707475; J. A. Casas and S. Dimopoulos, Phys. Lett. B 387, 107 (1996) [arXiv:hep-ph/9606237].

[21] J. A. Aguilar-Saavedra et al., arXiv:hep-ph/0511344.

[22] A. De Roeck, J. R. Ellis, F. Gianotti, F. Moortgat, K. A. Olive and L. Pape, arXiv:hep-ph/0508198.

[23] V. Berezinsky et al., Astropart. Phys. 5, 1 (1996) [arXiv:hepph/9508249]; M. Drees et al., Phys. Rev. D 56, 276 (1997) [Erratumibid. D 64, 039901 (2001)] [arXiv:hep-ph/9701219]; P. Nath and R. Arnowitt, Phys. Rev. D 56, 2820 (1997) [arXiv:hep-ph/9701301]; A. Bottino, F. Donato, N. Fornengo and S. Scopel, Phys. Rev. D 63, 125003 (2001) [arXiv:hep-ph/0010203]; D. G. Cerdeno and C. Munoz, JHEP 0410, 015 (2004) [arXiv:hep-ph/0405057]; H. Baer, A. Mustafayev, S. Profumo, A. Belyaev and X. Tata, JHEP 0507, 065 (2005) [arXiv:hep-ph/0504001]; J. R. Ellis, K. A. Olive and Y. Santoso, Phys. Lett. B 539, 107 (2002) [arXiv:hep-ph/0204192]; J. R. Ellis, T. Falk, K. A. Olive and Y. Santoso, Nucl. Phys. B 652, 259 (2003) [arXiv:hep-ph/0210205]; A. De Roeck et al., arXiv:hep-ph/0508198.

[24] L. S. Stark, P. Hafliger, A. Biland and F. Pauss, JHEP 0508, 059 (2005) [arXiv:hep-ph/0502197].

[25] H. Baer, A. Belyaev, T. Krupovnickas and A. Mustafayev, JHEP 0406 (2004) 044 [arXiv:hep-ph/0403214]. 
[26] T. Ibrahim and P. Nath, Phys. Rev. D 57 (1998) 478 [Erratum-ibid. D 58 (1998 ERRAT,D60,079903.1999 ERRAT,D60,119901.1999) 019901] [arXiv:hep-ph/9708456].

[27] T. Falk, K. A. Olive, M. Pospelov and R. Roiban, Nucl. Phys. B 560 (1999) 3 [arXiv:hep-ph/9904393].

[28] J. Hisano, M. Kakizaki, M. Nagai and Y. Shimizu, Phys. Lett. B 604 (2004) 216 [arXiv:hep-ph/0407169].

[29] M. Pospelov and A. Ritz, Phys. Rev. D 63 (2001) 073015 [arXiv:hep$\mathrm{ph} / 0010037]$.

[30] J. Hisano and Y. Shimizu, Phys. Rev. D 70 (2004) 093001 [arXiv:hep$\mathrm{ph} / 0406091]$.

[31] H. Leutwyler, Nucl. Phys. Proc. Suppl. 94 (2001) 108 [arXiv:hepph/0011049]; H. Leutwyler, Phys. Lett. B 378 (1996) 313 [arXiv:hep$\mathrm{ph} / 9602366]$.

[32] D. Chang, W. Y. Keung and A. Pilaftsis, Phys. Rev. Lett. 82, 900 (1999) [Erratum-ibid. 83, 3972 (1999)] [arXiv:hep-ph/9811202].

[33] G. Belanger, F. Boudjema, S. Kraml, A. Pukhov and A. Semenov, arXiv:hep-ph/0604150.

[34] C. Lee, V. Cirigliano and M. J. Ramsey-Musolf, Phys. Rev. D 71 (2005) 075010 [arXiv:hep-ph/0412354].

[35] M. Carena et al., Nucl. Phys. B 503 (1997) 387 [arXiv:hep-ph/9702409]; M. Carena et al., Nucl. Phys. B 599 (2001) 158 [arXiv:hep-ph/0011055].

[36] S. Profumo, private correspondence. 
[37] O. Lebedev, K. A. Olive, M. Pospelov and A. Ritz, Phys. Rev. D 70, 016003 (2004) [arXiv:hep-ph/0402023].

[38] T. Gajdosik, R. M. Godbole and S. Kraml, JHEP 0409 (2004) 051 [arXiv:hep-ph/0405167]; A. Dedes and S. Moretti, Phys. Rev. Lett. 84 (2000) 22 [arXiv:hep-ph/9908516]; G. L. Kane and L. T. Wang, Phys. Lett. B 488 (2000) 383 [arXiv:hep-ph/0003198]; A. Arhrib, D. K. Ghosh and O. C. W. Kong, Phys. Lett. B 537 (2002) 217 [arXiv:hep-ph/0112039]; M. Carena, J. R. Ellis, S. Mrenna, A. Pilaftsis and C. E. M. Wagner, Nucl. Phys. B 659 (2003) 145 [arXiv:hepph/0211467]; M. Carena et al., Nucl. Phys. B 659 (2003) 145 [arXiv:hepph/0211467]; E. Christova, H. Eberl, W. Majerotto and S. Kraml, Nucl. Phys. B 639 (2002) 263 [Erratum-ibid. B 647 (2002) 359] [arXiv:hepph/0205227]; JHEP 0212 (2002) 021 [arXiv:hep-ph/0211063].

[39] S. Y. Choi et al., Eur. Phys. J. C 14 (2000) 535 [arXiv:hep-ph/0002033]; S. Y. Choi, M. Guchait, J. Kalinowski and P. M. Zerwas, Phys. Lett. B 479 (2000) 235 [arXiv:hep-ph/0001175]; S. Y. Choi et al., Eur. Phys. J. C 8 (1999) 669 [arXiv:hep-ph/9812236]; S. Y. Choi, M. Drees and B. Gaissmaier, Phys. Rev. D 70 (2004) 014010 [arXiv:hepph/0403054]; J. L. Kneur and G. Moultaka, Phys. Rev. D 61 (2000) 095003 [arXiv:hep-ph/9907360]; V. D. Barger, T. Han, T. J. Li and T. Plehn, Phys. Lett. B 475 (2000) 342 [arXiv:hep-ph/9907425].

[40] O. Kittel, arXiv:hep-ph/0504183.

[41] A. Bartl, T. Kernreiter and O. Kittel, Phys. Lett. B 578 (2004) 341 [arXiv:hep-ph/0309340]. 


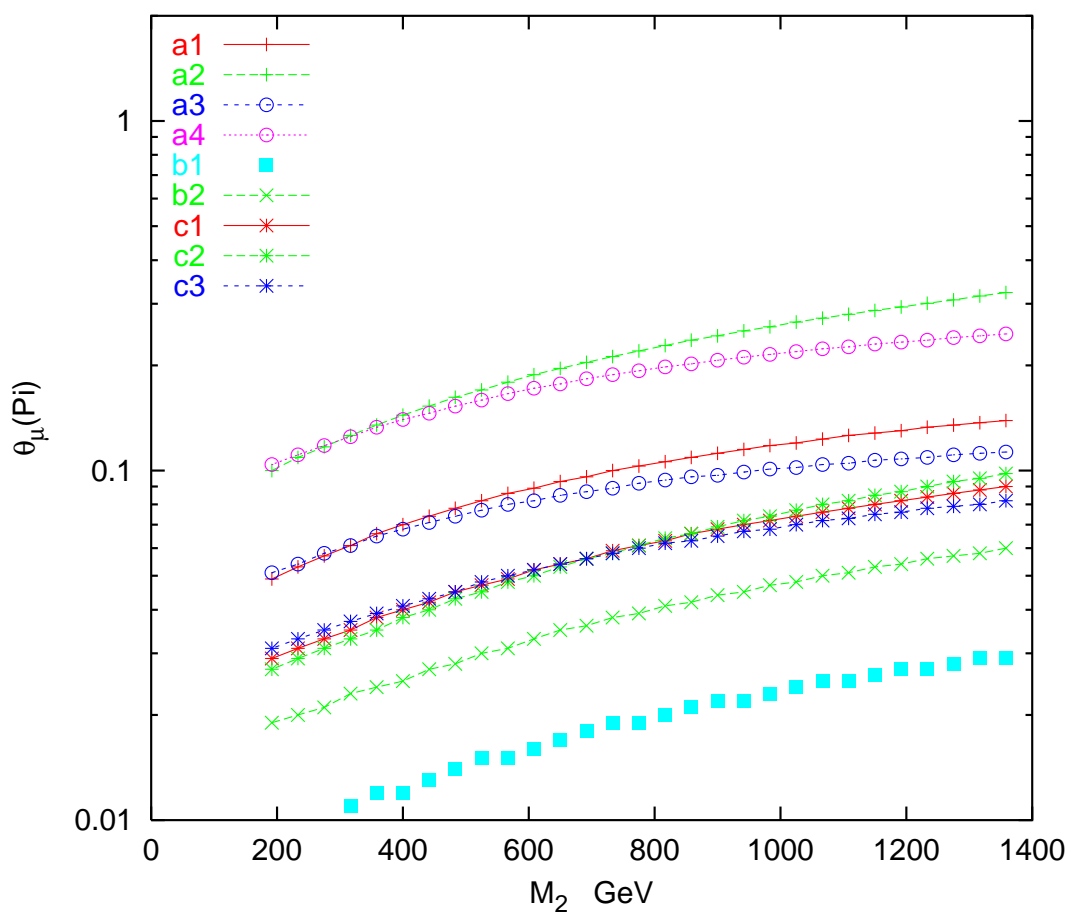

Figure 1: Maximum values of $\theta_{\mu}$ for which cancelation between contributions of the phases of $A_{e}$ and $\mu$ to $d_{e}$ is possible. For curves (a1-a2-a3-a4), $\tan \beta=$ $10,|\mu|=440 \mathrm{GeV}, m_{\tilde{e}_{L}}=305 \mathrm{GeV}, m_{\tilde{e}_{R}}=175 \mathrm{GeV}, m_{\tilde{\nu}_{L}}=295 \mathrm{GeV}$ and $M_{1}=155 \mathrm{GeV}$. The values of $\left|A_{e}\right|$ and $|\mu|$ for these curves are as follows: a1) $\left|A_{e}\right|=2000 \mathrm{GeV}$ and $|\mu|=440 \mathrm{GeV}$; a2) $\left|A_{e}\right|=4000 \mathrm{GeV}$ and $|\mu|=$ $440 \mathrm{GeV}$; a3) $\left|A_{e}\right|=2000 \mathrm{GeV}$ and $|\mu|=550 \mathrm{GeV}$; a4) $\left|A_{e}\right|=4000 \mathrm{GeV}$ and $|\mu|=550 \mathrm{GeV}$. For curves (b1) and (b2), $\tan \beta=20,|\mu|=500 \mathrm{GeV}, m_{\tilde{e}_{L}}=$ $450 \mathrm{GeV}, m_{\tilde{e}_{R}}=345 \mathrm{GeV}, m_{\tilde{\nu}_{L}}=440 \mathrm{GeV}$ and $M_{1}=175 \mathrm{GeV}$. For (b1), $\left|A_{e}\right|=2000 \mathrm{GeV}$ while for (b2) $\left|A_{e}\right|=4000 \mathrm{GeV}$. For (c1-c2-c3), $\tan \beta=10$, $m_{\tilde{e}_{L}}=405 \mathrm{GeV}, m_{\tilde{e}_{R}}=255 \mathrm{GeV}, m_{\tilde{\nu}_{L}}=400 \mathrm{GeV}, M_{1}=195 \mathrm{GeV}$ and $\left|A_{e}\right|=2000 \mathrm{GeV}$. The values of $|\mu|$ for curves (c1), (c2) and (c3) correspond to $|\mu|=500 \mathrm{GeV},|\mu|=400 \mathrm{GeV}$ and $|\mu|=600 \mathrm{GeV}$, respectively. 


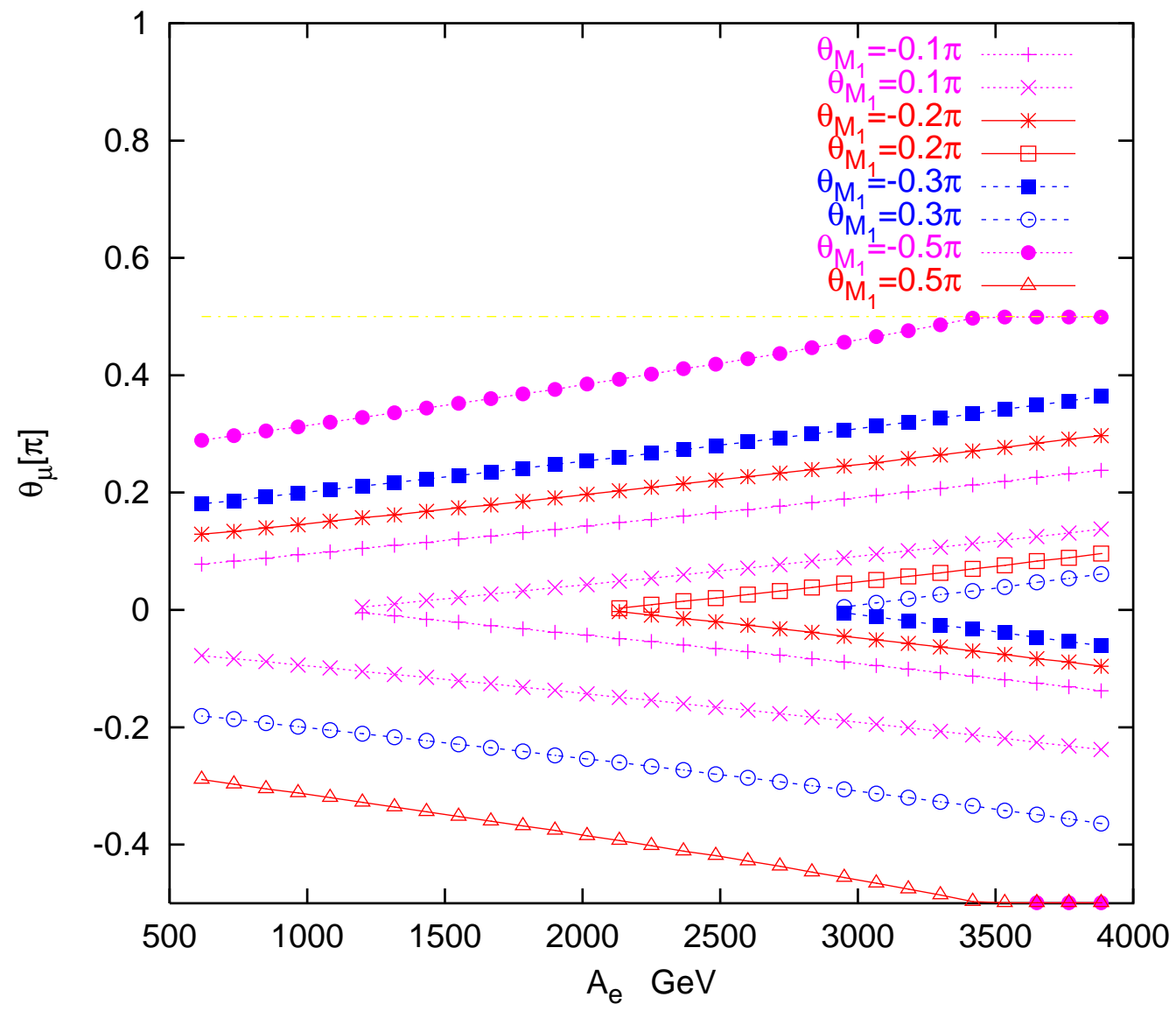

Figure 2: Maximum and minimum values of $\theta_{\mu}$ for which cancelation among the contributions of the phases of $M_{1}, A_{e}$ and $\mu$ is possible. Each curve corresponds to a different value of $\theta_{M_{1}}$ as indicated. We have taken $m_{\tilde{e}_{L}}=$ $190 \mathrm{GeV}, m_{\tilde{e}_{R}}=125 \mathrm{GeV}, m_{\tilde{\nu}}=295 \mathrm{GeV},\left|M_{1}\right|=97 \mathrm{GeV}, M_{2}=200 \mathrm{GeV}$, $\tan \beta=10$ which correspond to the values of these parameter for the SPS1a' benchmark [21]. However, the value of $|\mu|$ deviates from that of SPS1a': $|\mu|=440 \mathrm{GeV}$. 


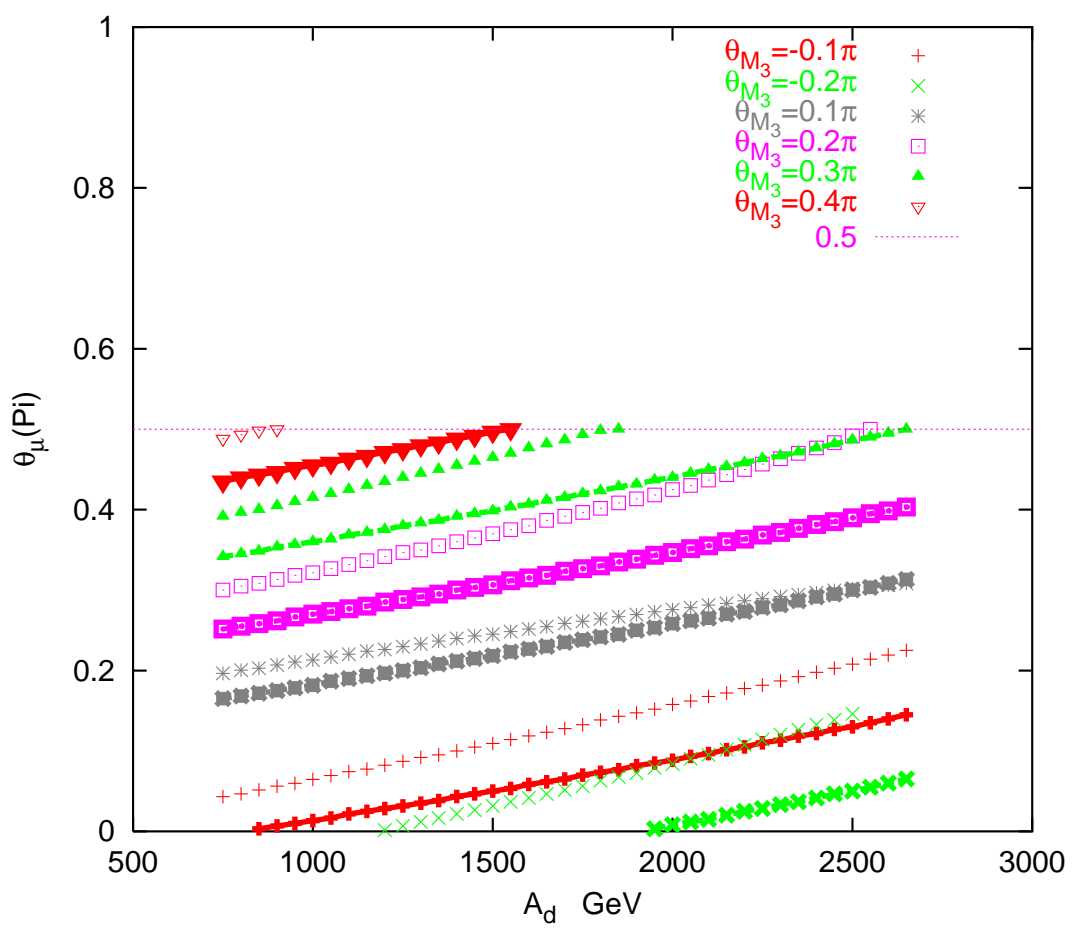

Figure 3: Maximum values of $\theta_{\mu}$ for which cancelation among the contributions of the phases of $A_{d}, A_{s}, M_{3}$ and $\mu$ to $d_{H g}$ is possible. We have taken $\left|A_{u}\right|=300 \mathrm{GeV},\left|A_{s}\right|=3000 \mathrm{GeV}, m_{d}=6.3 \mathrm{MeV}, m_{s}=119 \mathrm{MeV}$ and $m_{u}=3 \mathrm{MeV}$. Drawing the curves shown by discrete thin points the formula (8) is used while for the rest of the curves (thick points connected with lines) we have used Eq. 9. We have taken $|\mu|=440 \mathrm{GeV}$ while the rest of parameters correspond to the SPS1a' benchmark [21]: $m_{\tilde{d}_{L}}=m_{\tilde{s}_{L}}=565 \mathrm{GeV}$, $m_{\tilde{d}_{R}}=m_{\tilde{s}_{R}}=547 \mathrm{GeV}, M_{1}=97 \mathrm{GeV}, M_{2}=200 \mathrm{GeV},\left|M_{\tilde{g}}\right|=607 \mathrm{GeV}$ and $\tan \beta=10$. 


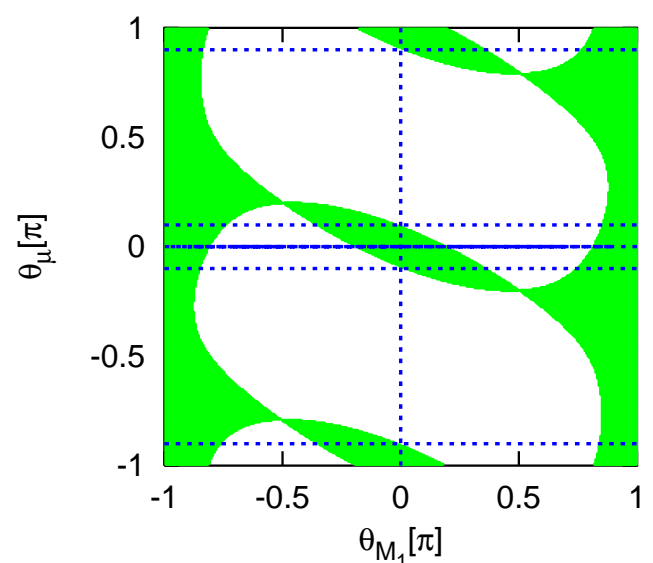

(a)

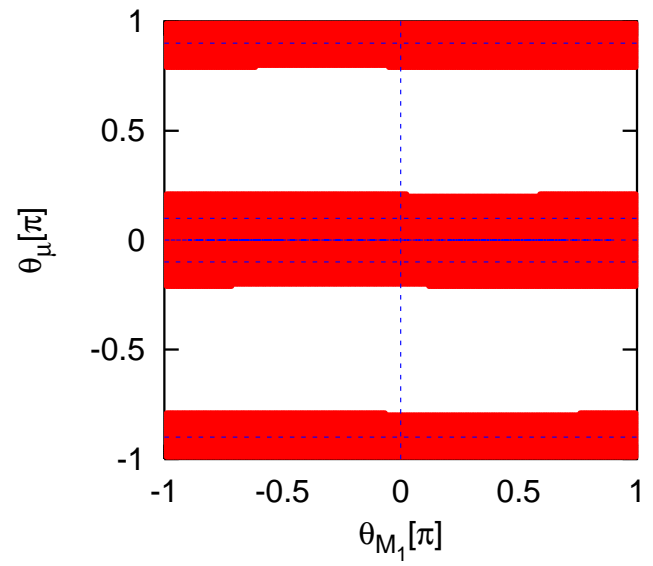

(c)

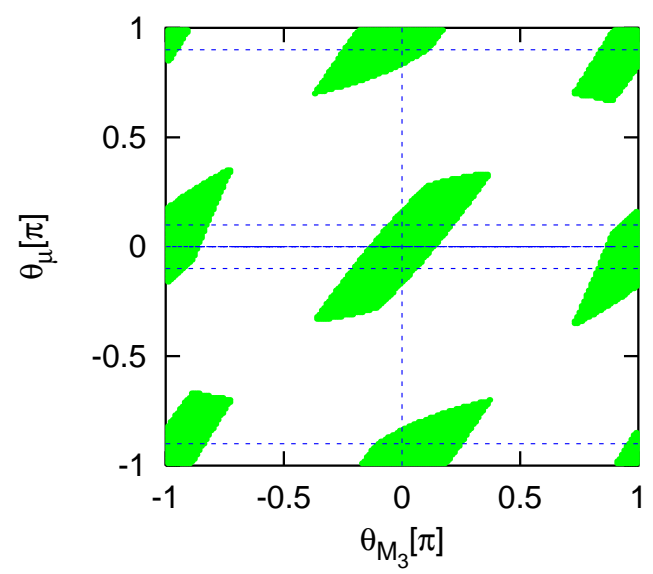

(b)

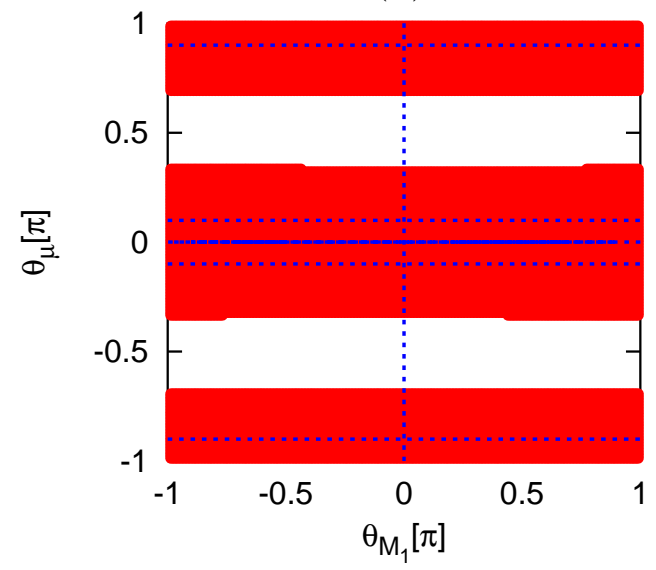

(d)

Figure 4: Shadowed areas depict the range of phases for which cancelation is possible. We have set $|\mu|=400 \mathrm{GeV}$ and the sfermion and gaugino masses correspond to their values for the SPS1a' benchmark [21] as explained in Figs. $(2,3)$. The horizontal dotted lines correspond to $\theta_{\mu}=-0.9 \pi,-0.1 \pi, 0.1 \pi$ and $0.9 \pi$. a) The range of $\theta_{\mu}-\theta_{M_{1}}$ for which cancelation between contributions of the phases of $A_{e}, \mu$, and $M_{1}$ can yield zero $d_{e}$. We have set $\left|A_{e}\right|=2000 \mathrm{GeV}$. b) Region where cancelation between contributions of the phases of the $A$ terms, $\mu$ and $M_{3}$ can yield $d_{n}=d_{H g}=0$. We have set $\theta_{M_{1}}=0$ and taken $\left|A_{u}\right|=300 \mathrm{GeV},\left|A_{s}\right|=\left|A_{d}\right|=3000 \mathrm{GeV}$. c) The same as (b) except that here we have set $\theta_{M_{3}}=0$. d) The same as (b,c) except that here all phases $\left(\theta_{M_{1}}, \theta_{M_{3}}, \theta_{\mu}, \theta_{A_{u}}, \theta_{A_{d}}\right.$ and $\left.\theta_{A_{s}}\right)$ are allowed to vary. 


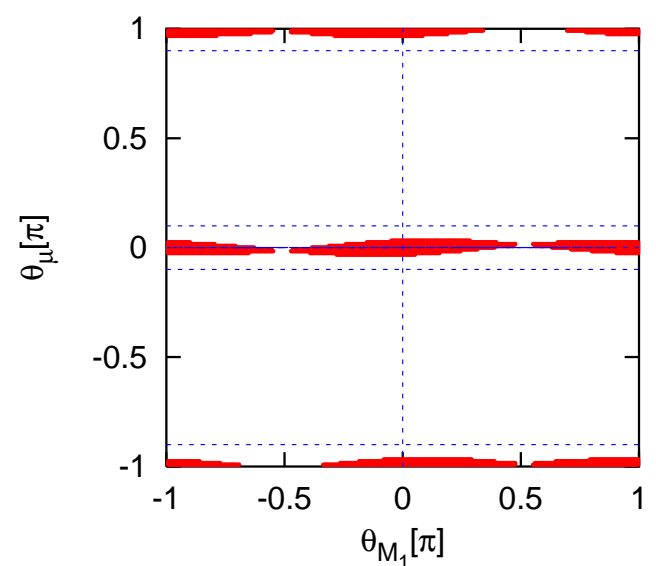

(a)

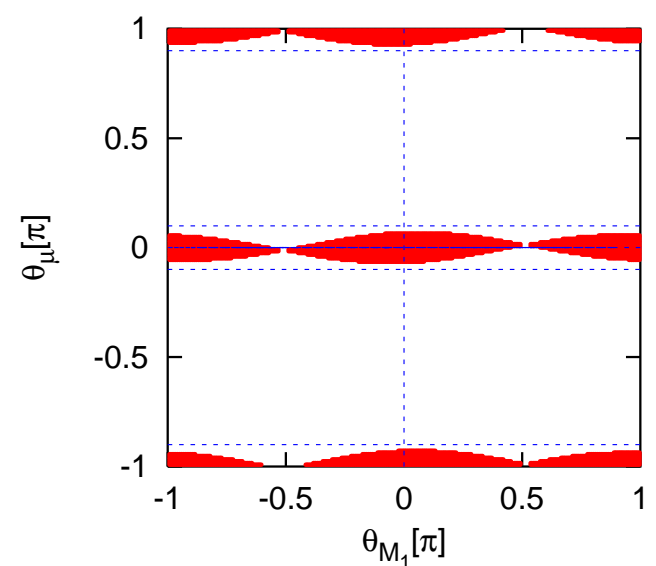

(b)

Figure 5: The shadowed areas show the values of phases for which different contributions to $d_{e}$ can cancel each other. The horizontal dotted lines correspond to $\theta_{\mu}=-0.9 \pi,-0.1 \pi, 0.1 \pi$ and $0.9 \pi$. We have chosen input parameters such that the Bino-Higgsino mixing is sizeable: a) $|\mu|=200 \mathrm{GeV}$, $\left|M_{1}\right|=150 \mathrm{GeV}, M_{2}=312 \mathrm{GeV}, m_{\tilde{e}_{L}}=336 \mathrm{GeV}, m_{\tilde{e}_{R}}=223 \mathrm{GeV}$, $m_{\tilde{\nu}_{L}}=327 \mathrm{GeV},\left|A_{e}\right|=2000 \mathrm{GeV}$ and $\tan \beta=10$; b) the same as (a) except $\left|A_{e}\right|=4000 \mathrm{GeV}$. 


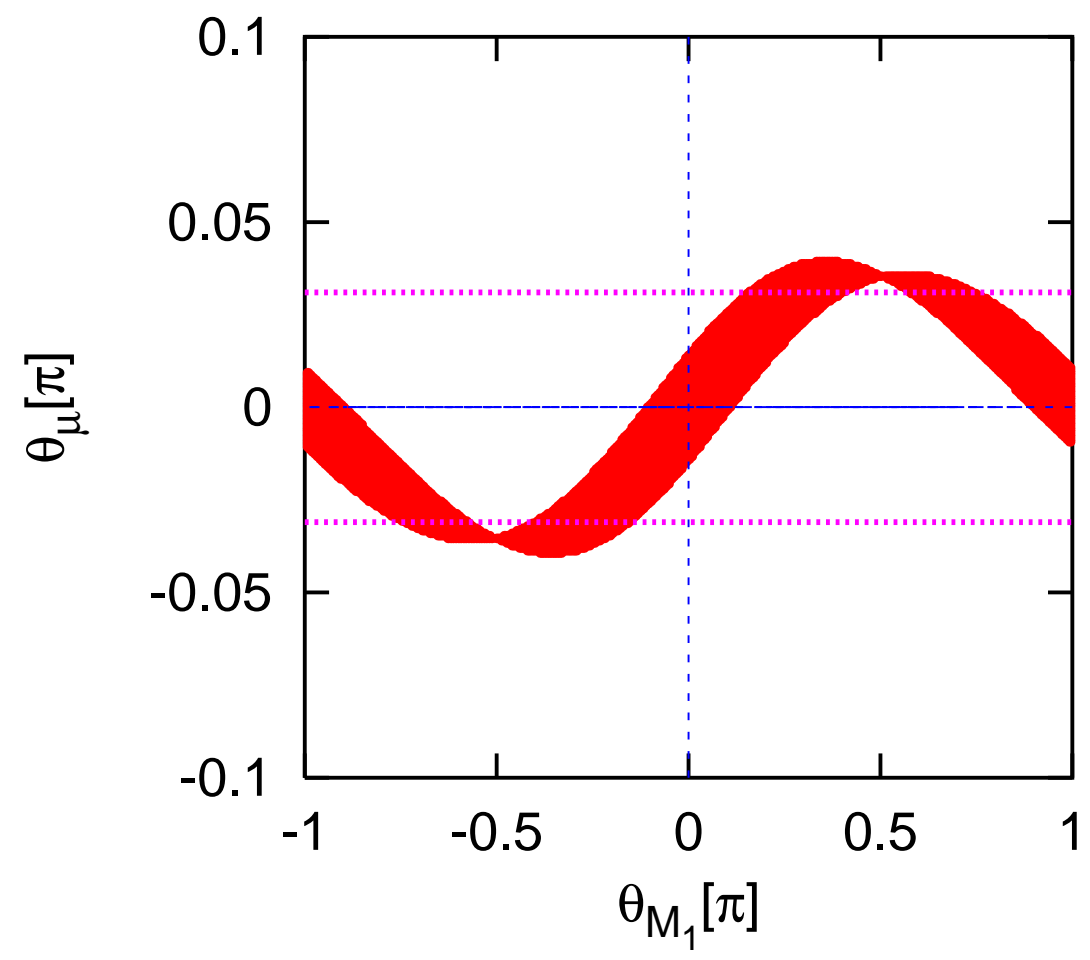

Figure 6: The range of phases of $\mu$ and $M_{1}$ for which total cancelation among the contributions of the phases $\mu, M_{1}$ and $A_{e}$ to $d_{e}$ is possible. We have taken $m_{\tilde{e}_{L}}=392 \mathrm{GeV}, m_{\tilde{e}_{R}}=220 \mathrm{GeV}, m_{\tilde{\nu}_{L}}=385 \mathrm{GeV},\left|A_{e}\right|=$ $700 \mathrm{GeV},\left|M_{1}\right|=200 \mathrm{GeV}$ and $M_{2}=415 \mathrm{GeV}$ and $\tan \beta=10$. We have set $|\mu|=200 \mathrm{GeV}=\left|M_{1}\right|$ which corresponds to the neutralino-driven resonance condition of electroweak baryogenesis. The purple dotted horizontal lines depict $\sin \theta_{\mu}= \pm 0.1$. 


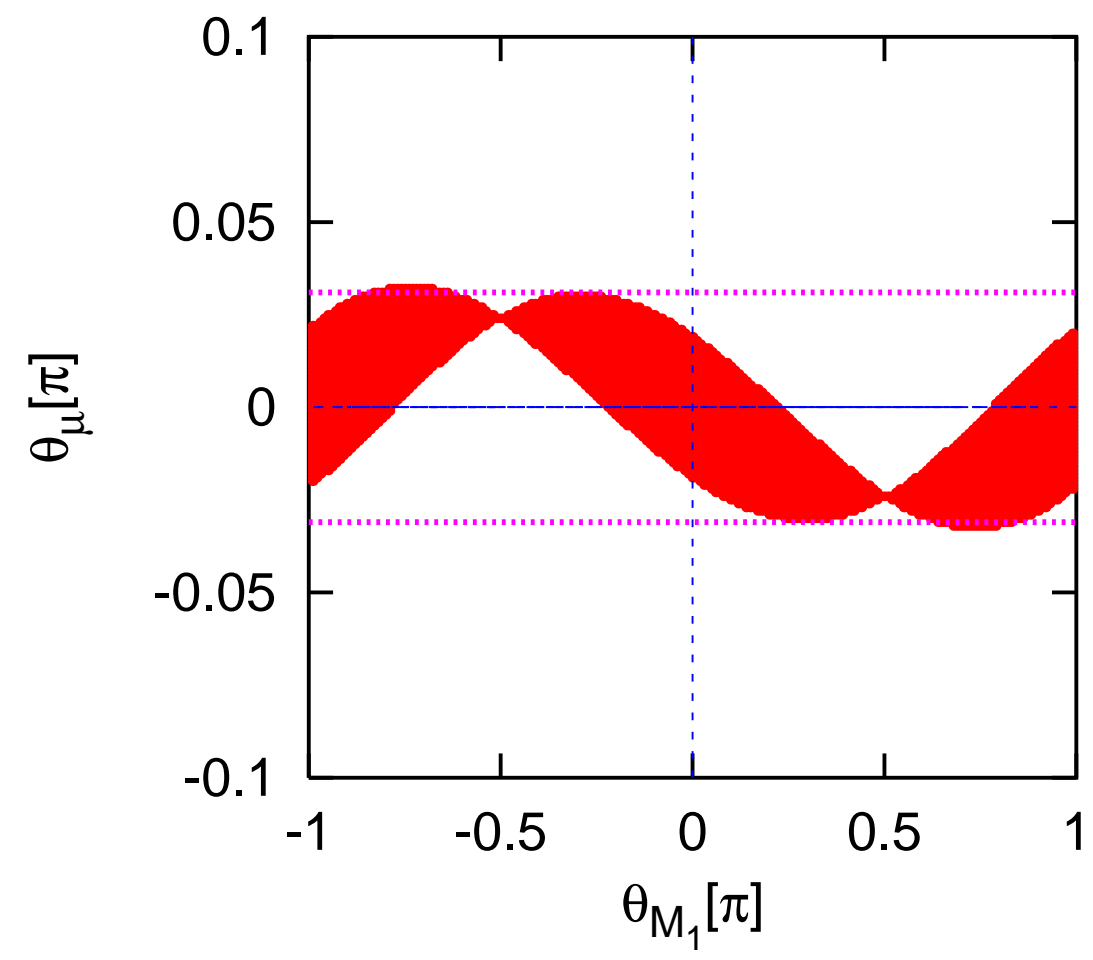

Figure 7: The range of phases of $\mu$ and $M_{1}$ for which total cancelation among the contributions of the phases $\mu, M_{1}$ and $A_{e}$ to $d_{e}$ is possible. We have taken $m_{\tilde{e}_{L}}=333 \mathrm{GeV}, m_{\tilde{e}_{R}}=187 \mathrm{GeV}, m_{\tilde{\nu}_{L}}=324 \mathrm{GeV},\left|A_{e}\right|=$ $700 \mathrm{GeV},\left|M_{1}\right|=167 \mathrm{GeV}$ and $M_{2}=348 \mathrm{GeV}$ and $\tan \beta=10$. We have set $|\mu|=340 \mathrm{GeV} \simeq M_{2}$ which corresponds to the chargino-driven resonance condition of electroweak baryogenesis. The purple dotted horizontal lines depict $\sin \theta_{\mu}= \pm 0.1$. 


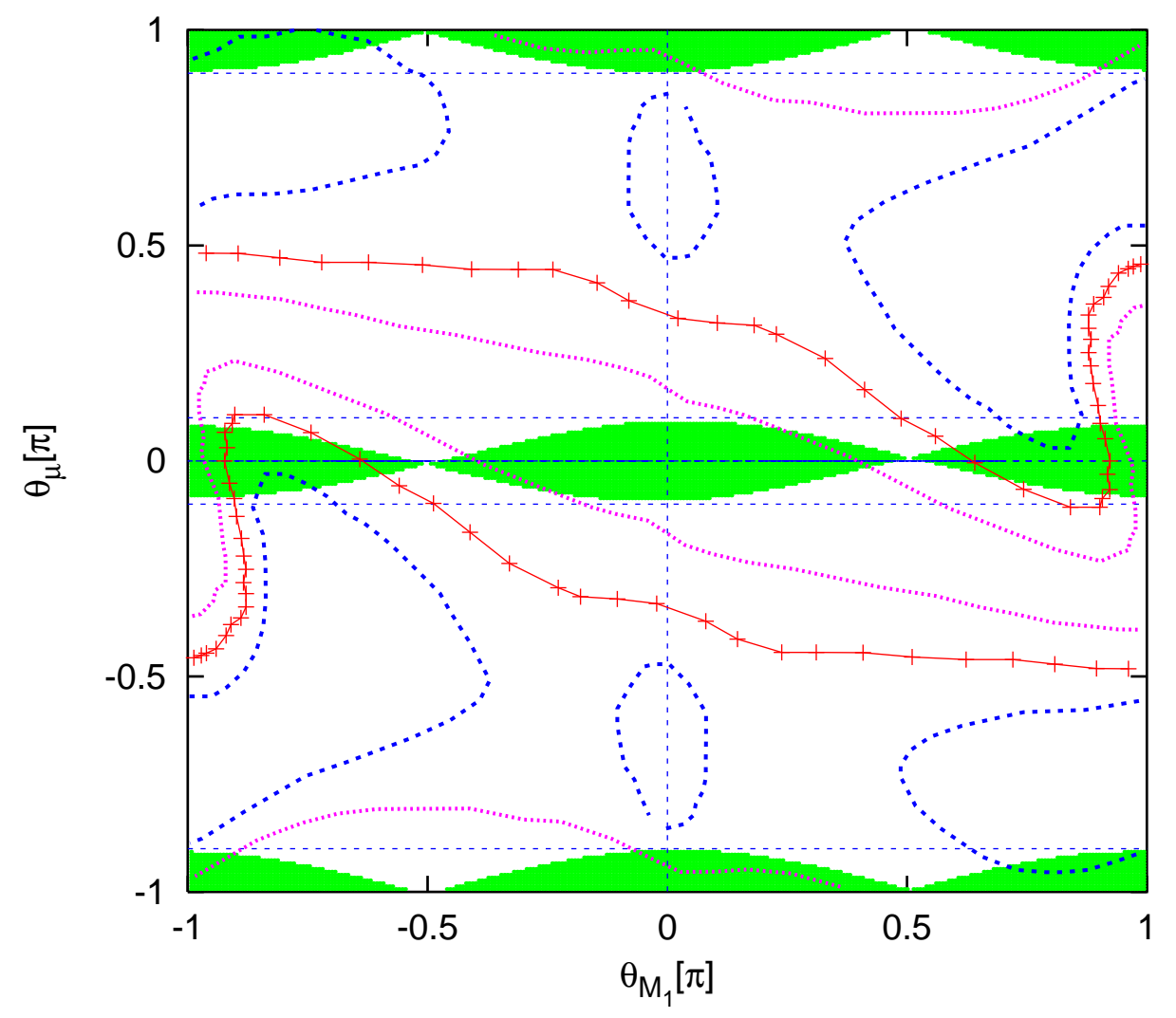

Figure 8: Shadowed areas show the region where cancelation can yield vanishing $d_{e}$. The curves, which are borrowed from Fig. 2.12.b of [40], correspond to various values of $A_{C P}$ (see the text for the definition of $A_{C P}$ ): Dashed lines correspond to $A_{C P}= \pm 45 \%$; curves marked with + indicate $A_{C P}= \pm 30 \%$ and finally the pink curves correspond to $A_{C P}= \pm 15 \%$. To draw the shadowed area we have used the same input parameters as in Fig 2.12.b of [40]: $|\mu|=300 \mathrm{GeV}, m_{\tilde{e}_{L}}=378 \mathrm{GeV}, m_{\tilde{e}_{R}}=211 \mathrm{GeV}, m_{\tilde{\nu}_{L}}=370 \mathrm{GeV}$, $\left|M_{1}\right|=192 \mathrm{GeV}, M_{2}=400 \mathrm{GeV},\left|A_{e}\right|=2000 \mathrm{GeV}$ and $\tan \beta=5$. The horizontal dotted lines correspond to $\theta_{\mu}=-0.9 \pi,-0.1 \pi, 0.1 \pi$ and $0.9 \pi$. 